\title{
Recurrent radiation-associated leiomyosarcoma after postoperative radiotherapy for breast invasive ductal carcinoma: a case report and literature review
}

\author{
Wei Zhang ${ }^{1}$, Xinlan $\mathbf{Q u}^{2}$, Yuan Fang ${ }^{3}$ \\ ${ }^{1}$ Department of Radiation and Medical Oncology, Zhongnan Hospital of Wuhan University, Wuhan, China; ${ }^{2}$ Department of Gynaecology and \\ Obstetrics, Zhongnan Hospital of Wuhan University, Wuhan, China; ${ }^{3}$ Department of Thyroid \& Mammary Gland Surgery, Zhongnan Hospital of \\ Wuhan University, Wuhan, China \\ Correspondence to: Dr. Yuan Fang. Department of Thyroid \& Mammary Gland Surgery, Zhongnan Hospital of Wuhan University, 169 Donghu Road, \\ Wuhan 430071, China. Email: fy_whu@126.com.
}

\begin{abstract}
Radiation-associated leiomyosarcoma has a poor prognosis. Fewer than 20 cases of radiationassociated leiomyosarcoma of the breast have been reported in the literature to date. We report the first case of recurrent radiation-associated leiomyosarcoma after invasive ductal carcinoma postoperative radiotherapy. A 45-year-old female patient with no familial history of cancer underwent breast-conserving surgery (BCS) and dissection of axillary lymph nodes for invasive ductal carcinoma in the right upper outer region in 2014. After the operation, she received adjuvant chemotherapy followed by radiotherapy and endocrine therapy. She came to our institution for a lump in the right breast radiation region in 2019. Ultrasonography revealed an oval mass, with ill-defined borders, irregular margins, and speculation at 6 o'clock direction of the right breast, approximately $2.5 \mathrm{~cm}$ from the nipple. Ultimately, excisional biopsy established the pathological diagnosis of leiomyosarcoma. The female then underwent simple mastectomy of the right breast. The patient received no radiation or chemotherapy after the simple mastectomy. After 13 months of follow-up, a lump was found in the right chest wall and axillary levels of the radiation region. Excisional biopsy confirmed the pathological diagnosis of leiomyosarcoma. This rare case indicated that simple mastectomy seemed to be inadequate treatment for radiation-associated leiomyosarcoma.
\end{abstract}

Keywords! Radiation-associated sarcomas; radiation-associated leiomyosarcoma; radiotherapy; breast cancer; case report

Submitted Dec 22, 2020. Accepted for publication Mar 25, 2021.

doi: 10.21037/apm-20-2561

View this article at: http://dx.doi.org/10.21037/apm-20-2561

\section{Introduction}

Approximately half of patients with malignant cancer receive radiotherapy (RT) as part of their oncologic treatment (1). Although postoperative RT is well recognized to increase the overall survival (OS) rate of cancer patients, it may also lead to secondary malignancies due to the carcinogenic effect of ionizing radiation (2). Radiationassociated sarcomas (RAS), also known as radiation-induced sarcomas (RIS), are a rare type of secondary malignancy following RT, and occur in $<1 \%$ of patients treated with RT $(2,3)$. Radiation-associated leiomyosarcoma accounts for $8 \%$ of RAS patients (2). There is a long period between RT and RAS development, ranging from 3 to 20.3 years (4). $\mathrm{RT}$ is the main cause of RAS, and TP53 gene mutations are another risk factor. Breast cancer patients with pathogenic variants of TP53 have shorter intervals of RAS after radiotherapy (occasionally $2-3$ years) than patients with no pathogenic variants (usually approximately $9-15$ years) (5). The most common symptom of RAS is a painless mass. Therefore, this disease is easy to misdiagnose. Although recurrent sarcomas are common, recurrent radiationassociated leiomyosarcoma of the breast after breast 

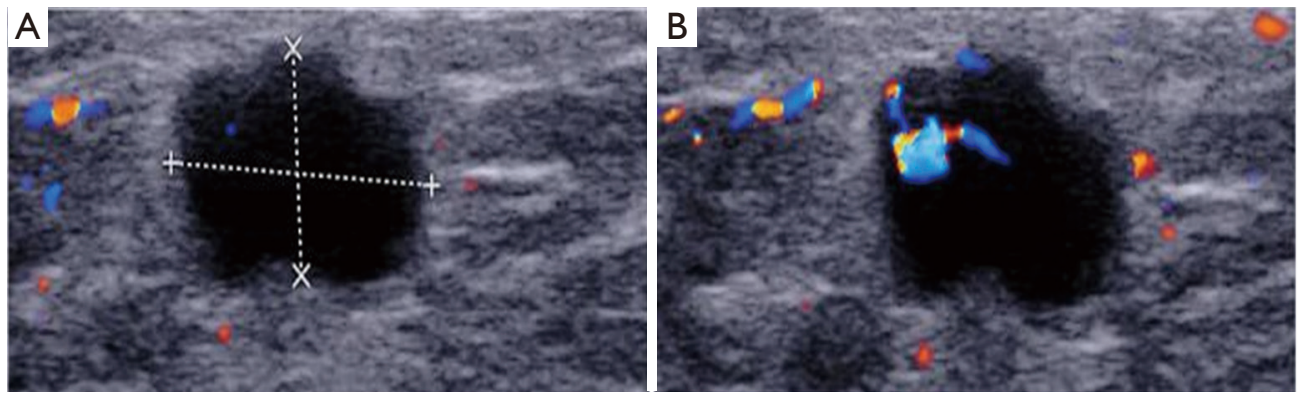

Figure 1 Ultrasound images. (A) Ultrasonography showing an oval, hypoechoic mass, $1.0 \mathrm{~cm} \times 0.7 \mathrm{~cm}$ in size, with ill-defined borders and irregular margins, and speculation at 6 o'clock direction of the right breast; (B) power Doppler US showing rich blood flow inside the tumour, and arterial blood flow was detected.

cancer radiotherapy has not been reported. In this case report, we present a case of recurrent radiation-associated leiomyosarcoma arising in the irradiated region after breast cancer radiotherapy for invasive ductal carcinoma. We present the following case in accordance with the CARE reporting checklist (available at http://dx.doi.org/10.21037/ apm-20-2561).

\section{Case presentation}

A 45-year-old female patient with no familial history of cancer underwent breast-conserving surgery (BCS) and dissection of axillary lymph nodes of the right breast upper outer region on March 4, 2014. Postoperative histopathology revealed an invasive ductal carcinoma $2.5 \mathrm{~cm} \times 2.0 \mathrm{~cm}$ in size, with approximately $1-\mathrm{cm}$ margins. The 18 axillary lymph nodes proved to be tumour free, as were the resection margins. Immunohistochemistry demonstrated that estrogen and progesterone receptors were positive (ER+/PR+), HER2 receptor was positive (HER2 3+), and the Ki67 staining positive rates were approximately $80 \%$. Therefore, the patient was classified as having luminal B subtype breast cancer. According to the UICC-pTNM classification, the clinicopathological class was stage IIA. Based on the above characteristics, the patient received adjuvant chemotherapy with fluorouracil-epirubicin-cyclophosphamide followed by docetaxel (FEC-T) plus human HER2-targeted therapy with trastuzumab for one year. After 6 cycles of chemotherapy, she received RT at $50 \mathrm{~Gy}$ in 25 fractions to the whole right breast, followed by $10 \mathrm{~Gy}$ in 5 fractions as a boost to the lumpectomy cavity. The clinical target volumes (CTVs) of the patient included the total right glandular breast tissue: the dorsal border was the outer rim of the ribs; the ventral border was $5 \mathrm{~mm}$ under the skin surface; the cranial border extended to the level of the caudal edge of the sternoclavicular joint; the caudal border was $2 \mathrm{~cm}$ below of the lower edge of the breast; the medial border extended to the sternal stem edge, no further than the midline of the sternum; and the lateral border extended to the right midaxillary line. Endocrine therapy began at the same time after chemotherapy, initially with tamoxifen for 2 years followed by conversion to letrozole until now due to bilateral oophorectomy for increased ovarian cysts in 2017. After breast surgery, the patient came regularly for postoperative screening. Physical examination, blood biochemistry, tumour markers, and colour ultrasound of the breast and lymph nodes were performed every 3 months. Mammography, chest computed tomography (CT), abdominal CT, bone scan and brain magnetic resonance imaging (MRI) were performed every 12 months. No recurrence was found for more than 5 years.

However, a lump was found located at the 6 o'clock position in the right breast on July 21, 2019. Physical examination revealed a firm mass, $1.0 \mathrm{~cm} \times 0.6 \mathrm{~cm}$ in size with an ill-circumscribed and painless mass, located in the radiation area. There was no nipple dimpling and no palpable axillary lymph node to indicate metastasis. Other results in her general physical examination were normal. Ultrasonography (Figure $1 A$ ) found an oval, hypoechoic mass, $1.0 \mathrm{~cm} \times 0.7 \mathrm{~cm}$ in size, with ill-defined borders and irregular margins, and speculation at 6 o'clock direction of the right breast, approximately $2.5 \mathrm{~cm}$ from the nipple. Power Doppler US (Figure 1B) showed arterial blood flow inside the tumour, which was assessed as category 5 . Biochemical blood examination, including tumour markers, revealed no abnormalities. Therefore, it was necessary to perform excisional biopsy. Postoperative histopathology (Figure 2) revealed a $1.0 \mathrm{~cm} \times 0.8 \mathrm{~cm}$ tumour with typical spindle cells arranged as long fascicles in a collagenous background. The 
tumour was well defined, well encapsulated, and composed of binucleated and multinucleated cells. Immunohistochemistry (Figure 3) demonstrated that the tumour cells were positive for SMA, calsdesmon, b-catenin, desmin, and Ki67 (30\%), but negative for CK, CAM5.2, GATA-3, P63, and S-100, supporting the diagnosis of leiomyosarcoma. The patient was then submitted to simple mastectomy of the right breast with approximately $3-\mathrm{cm}$ margins, including the nipple, areola, breast gland and fascia of the pectoralis major muscle. Postoperative pathology showed that all margins were negative.

The patient received no radiation or chemotherapy after the simple mastectomy, but she came to our hospital

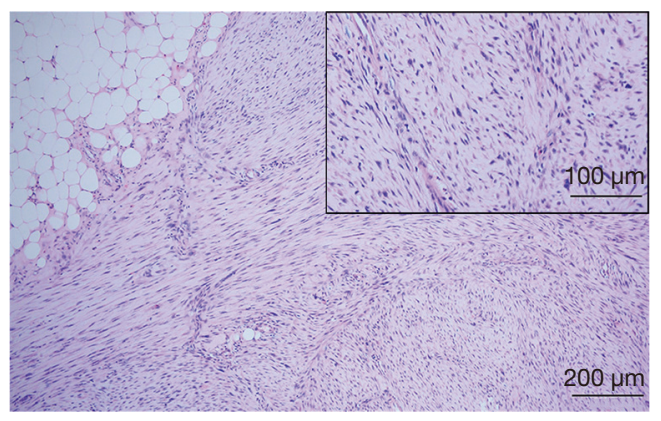

Figure 2 Histopathology revealed typical spindle cells arranged as long fascicles in a collagenous background. The tumour was well defined, well encapsulated, and composed of a pack of binucleated and multinucleated cells. for postoperative screening regularly. After 13 months of follow-up, a painless lump was found located in the right chest wall of the radiation region and axillary levels. The patient underwent ultrasonography (Figure $4 A$ ), which revealed a hypoechoic mass with a clear boundary and heterogeneous echo. The lump was located subcutaneously in the right chest wall and was approximately $0.3 \mathrm{~cm} \times$ $0.2 \mathrm{~cm}$ in size, approximately $0.1 \mathrm{~cm}$ away from the body surface. Power Doppler US (Figure 4B) showed that arterial blood flow inside the tumour. Finally, excisional biopsy confirmed the pathological diagnosis of leiomyosarcoma on August 17, 2020. The patient was so disappointed with the recurrence of radiation-associated leiomyosarcoma that she refused to undergo a second extended surgical resection. The timeline is shown in Figure 5.

All procedures performed in studies involving human participants were in accordance with the ethical standards of the institutional and/or national research committee(s) and with the Helsinki Declaration (as revised in 2013). Written informed consent was obtained from the patient.

\section{Discussion}

Primary leiomyosarcoma of the breast accounts for $<0.0006 \%$ of all breast malignancies, and fewer than 80 cases have been reported in the literature to date (6). Additionally, fewer than 20 cases of radiation-associated leiomyosarcoma of the breast have been reported in the literature to date. Here we report the first case of recurrent radiation-associated
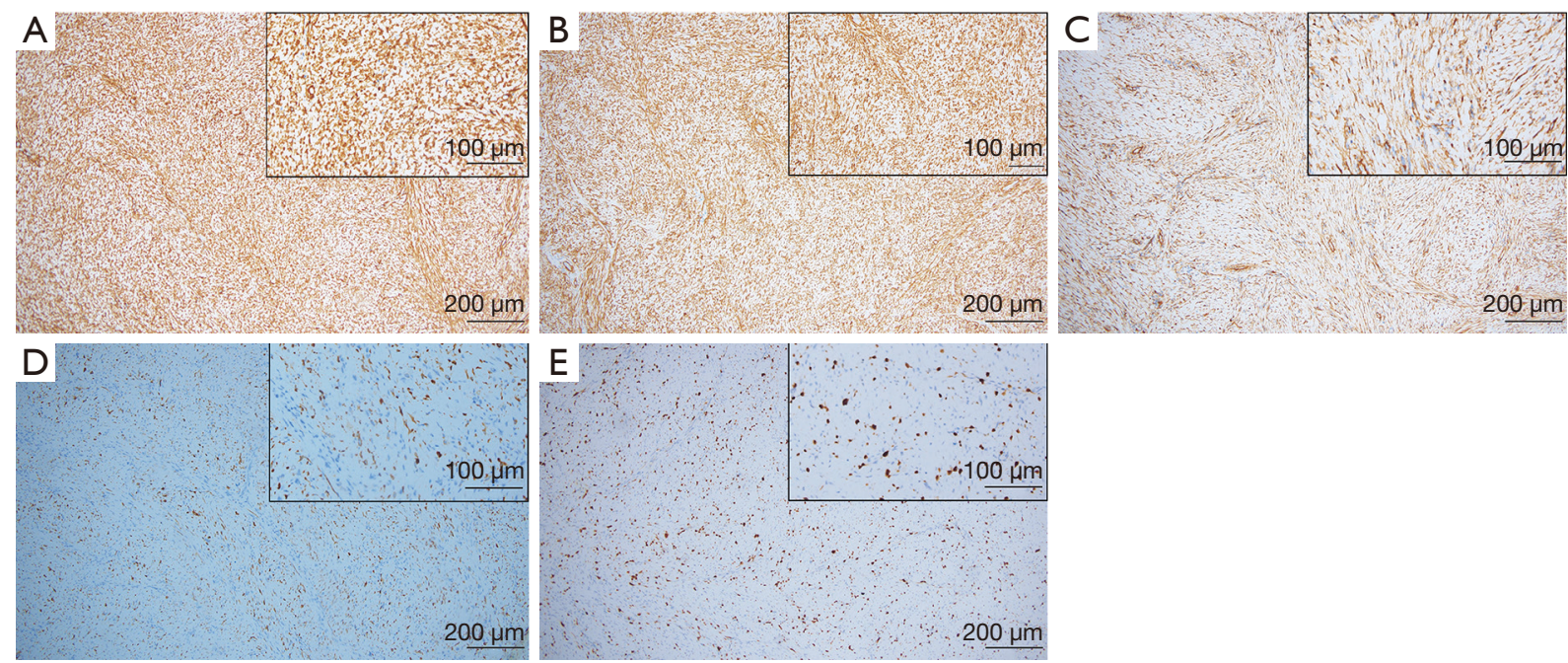

Figure 3 (A) Immunohistochemical features. Immunohistochemistry demonstrated the tumour cells were positive for SMA, (B) calsdesmon, (C) $\beta$-catenin, (D) desmin, (E) Ki67 (30\%). 

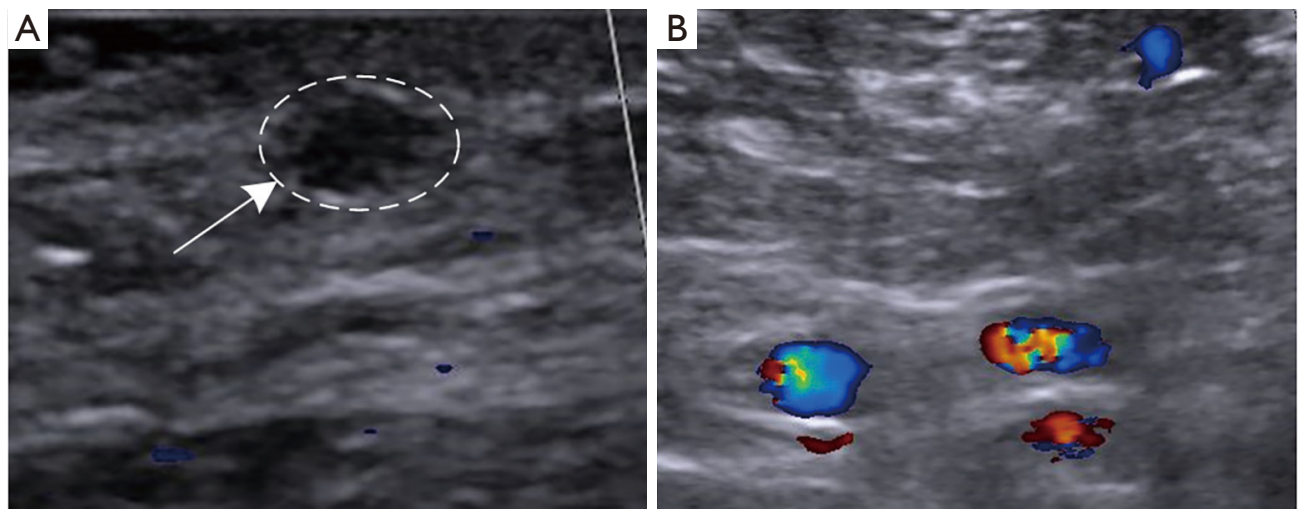

Figure 4 Ultrasound images. (A) Ultrasonography showing a hypoechoic mass with a clear boundary and heterogeneous echo (circle), located subcutaneously in the right chest wall and was approximately $0.3 \mathrm{~cm} \times 0.2 \mathrm{~cm}$ in size, approximately $0.1 \mathrm{~cm}$ away from the body surface. (B) Power Doppler US showing rich blood flow inside the tumor, and arterial blood flow was detected.

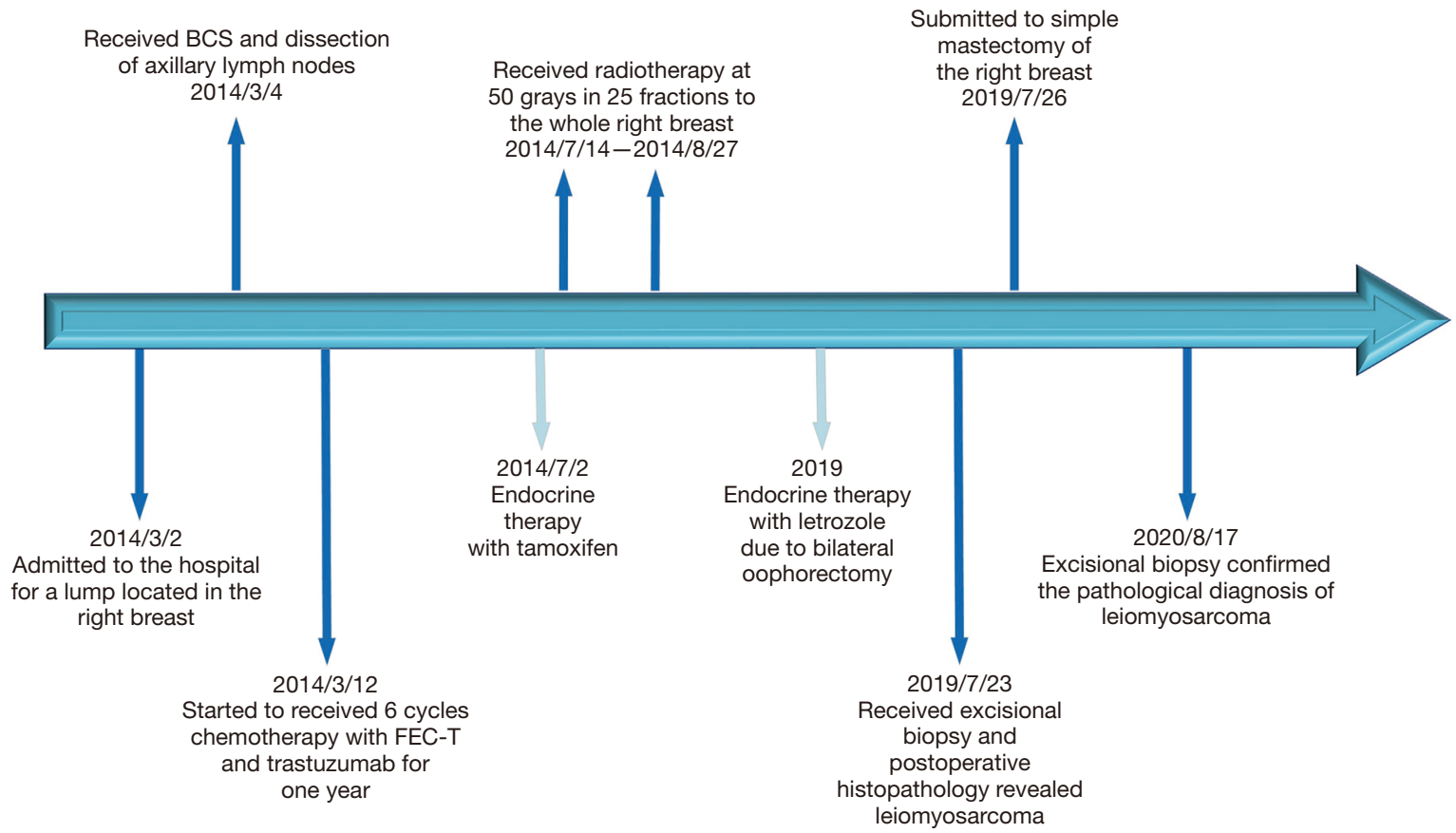

Figure 5 Timeline. The information of the patient with secondary leiomyosarcoma after breast cancer radiotherapy. FEC-T, fluorouracilepirubicin-cyclophosphamide followed by docetaxel; BCS, breast-conserving surgery.

leiomyosarcoma following invasive ductal carcinoma. Radiation-associated leiomyosarcoma is a rare type of RAS that accounts for $8 \%$ of RAS patients (2). RAS originates from mesenchymal tissue. It accounts for up to $5 \%$ of sarcomas but occurs in less than $1 \%$ of patients treated with RT (2,7). Radiation exposure among TP53 mutation carriers seems to increase the rate of second cancers. Studies have reported that the incidence of RAS in patients with pathogenic germline pathogenicity of TP53 is 16-33\% (5). With the increased use of RT and multidisciplinary therapy to improve the OS of patients with malignant tumours, the incidence of RAS is increasing (8). The current universally used diagnostic criteria of RAS were defined by Cahan et al.: (I) histologic confirmation of a sarcoma; (II) tumour arising within or adjacent to a previously irradiated field; and (III) evidence of an initial non-malignancy, or a malignant tumour 
different from secondary sarcoma (9).

Leiomyosarcoma usually arises in the uterus, abdominal and urologic viscera, and the walls of large and small blood vessels (10). Leiomyosarcoma of the breast is relatively rare. It may originate from the smooth muscle around the nipple-areola or blood vessels $(11,12)$. The transformation of myoepithelial cells and myofibroblasts may be another possible origin $(13,14)$. Radiation-associated leiomyosarcoma is too rare to conclusively describe the clinical features, diagnosis, and treatment characteristics. Here, we summarize the characteristics of RAS, as radiation-associated leiomyosarcoma accounts for $8 \%$ of RAS patients. The most common symptom of patients diagnosed with RAS is a painless mass. RAS usually occurs in women. The most common primary malignancy is breast carcinoma (55\%), including invasive carcinoma and ductal carcinoma in situ. In a previous study, 13 sarcomas were located in the breast, while others were located in the chest wall, sternum, supraclavicular area, scapula, and axilla among 35 RAS cases (4). In this case report, the patient developed radiation-associated leiomyosarcoma in the breast and chest wall.

Radiation dose and field are important factors in the development of RAS. RAS has occurred occasionally after a median dose of 50-55 Gy in 25-27 fractions in the whole breast, followed by a 10-26 Gy boost to the tumour or tumour bed when necessary $(4,15,16)$. In our case, the female patient's clinical and treatment characteristics were consistent with those of a previous study. She received RT at 50 Gy to the whole right breast, followed by a $10 \mathrm{~Gy}$ boost to the lumpectomy cavity. Her primary symptom was a painless mass, and the latency period was 5 years.

A definitive diagnosis of leiomyosarcoma depends on histopathology combined with immunohistochemical staining. Typical histological features of circumscription include high cellularity and fusiform spindle cells with blunt-ended nuclei (17). In leiomyosarcoma, tumour cells are immunoreactive for SMA, vimentin and desmin and negative for the epithelial marker S-100 and growth factor receptors $(18,19)$. In our case, the strong expression of SMA, in addition to the typical cellular morphology, indicates the smooth muscle origin of this tumour. Core needle biopsy (CNB) and excisional biopsy are recommended to diagnose leiomyosarcoma. However, excisional biopsy may have lower false negative rate in diagnosis, because CNB may not puncture malignant transformation tissue. Hayashi et al. reported a case, in which $\mathrm{CNB}$ of the lump suggested atrophic mammary glands. After 3 months, Re-CNB was performed, which confirmed leiomyosarcoma (20).

The main treatment of RAS is complete excision of the tumour. The National Comprehensive Cancer Network (NCCN) recommends a resection margin of $2 \mathrm{~mm}$ for ductal carcinoma in situ and no ink on tumours for invasive breast cancer. However, considering the recurrence pathology and the malignant characteristics of leiomyosarcoma, wider tumour margins of at least $2 \mathrm{~cm}$ are needed for conservative breast treatment, and a resection margin of $3 \mathrm{~cm}$ is preferred (6). Thijssens et al. reported that patients with R0 (microscopically negative) resection had a significantly better survival rate than patients with R1 (microscopically positive), R2 (macroscopically positive), or no resection $(\mathrm{P}<0.05)(8)$. Dissection of axillary lymph nodes may not be necessary, since axillary lymph node metastasis has never been reported in the previous literature (21). Haematogenous dissemination is the main route of metastasis. Simple and modified radical mastectomy are the most common surgical methods (11). However, our case indicated that simple mastectomy was inadequate treatment for radiation-associated leiomyosarcoma, since the tumour recurred in the chest wall. Mito et al. shared their experiences in preventing the local recurrence of radiation-associated breast angiosarcoma by removing the irradiated tissue as much as possible, including all or nearly all previously irradiated breast skin, with a resection field that extends from the clavicle to at least $1 \mathrm{~cm}$ below the inframammary crease and from mid-sternum to the anterior border of the latissimus dorsi muscle (2). After a second recurrence, the patient refused to undergo a second extended surgical resection because the radical surgical protocol was too aggressive. She insisted on continuous observation and regular follow-up.

Although RT and chemotherapy are effective in select cases, their association with improved a prognosis of RAS has not been validated. In addition, breast re-irradiation may increase the toxicity for higher cumulative lifetime doses to critical organs, such as the lungs and heart. Reirradiation after radical adjuvant radiotherapy can be considered only for selected patients after careful informed consent. In our case report, the patient developed RAS after radical adjuvant radiotherapy, suggesting possible TP53 gene mutations. Re-irradiation not only increases radiationrelated toxicities, such as rib fracture, radiation pneumonia and soft tissue necrosis, but also increases the risk of developing RAS again. In view of the patient in our case report with $\mathrm{R} 0$ resection and the lack of sufficient strong evidence to support the effectiveness of chemotherapy and RT for RAS patients, she received no radiation or 
chemotherapy after simple mastectomy in our institution.

Poor prognosis is associated with RAS from a historical perspective. It is necessary for the patient to come regularly for postoperative screening. Horton et al. recommended follow-up examinations with mammogram and chest CT to be performed every 6 months in years 1-7 and annually thereafter (6). Tumour grade, depth of infiltration and achievement of margin-negative resection were the 3 main factors affecting tumour prognosis (22). Mito et al. demonstrated that the 3-year OS rate after diagnosis of RAS was $74 \%$ and that the 5 -year OS rate after diagnosis of RAS was $68 \%$, revealing that the current survival rates were higher than historically reported rates (2).

\section{Conclusions}

We report a globally rare case of recurrent radiationassociated leiomyosarcoma after RT for breast cancer. Unfortunately, genetic testing was not performed because the patient's tumour molecular type was luminal $\mathrm{B}$, the patient had no familial history of cancer, the patient was 45 years old at the time of the initial diagnosis, and there were high self-paid expenses. There is a lack of strong evidence to support the effectiveness of chemotherapy and RT for RAS patients. Simple mastectomy is inadequate treatment for radiation-associated leiomyosarcoma. Since BCS and RT are standard treatments for breast cancer patients, it is necessary to be aware of the possible development of secondary leiomyosarcoma in the irradiated region. The existing treatment options are inadequate for radiationassociated leiomyosarcoma. Future studies may illustrate the mechanisms, may improve OS or may even prevent the development of RAS based on genetic susceptibility.

\section{Acknowledgments}

We would like to thank the patient and her family for the consent of the publication.

Funding: This study was supported by National Natural Science Foundation of China (81601281).

\section{Footnote}

Reporting Checklist: The authors have completed the CARE reporting checklist. Available at http://dx.doi.org/10.21037/ apm-20-2561

Peer Review File: Available at http://dx.doi.org/10.21037/ apm-20-2561

Conflicts of Interest: All authors have completed the ICMJE uniform disclosure form (available at http://dx.doi. org/10.21037/apm-20-2561). The authors have no conflicts of interest to declare.

Ethical Statement: The authors are accountable for all aspects of the work in ensuring that questions related to the accuracy or integrity of any part of the work are appropriately investigated and resolved. All procedures performed in studies involving human participants were in accordance with the ethical standards of the institutional and/or national research committee(s) and with the Helsinki Declaration (as revised in 2013). Written informed consent was obtained from the patient.

Open Access Statement: This is an Open Access article distributed in accordance with the Creative Commons Attribution-NonCommercial-NoDerivs 4.0 International License (CC BY-NC-ND 4.0), which permits the noncommercial replication and distribution of the article with the strict proviso that no changes or edits are made and the original work is properly cited (including links to both the formal publication through the relevant DOI and the license). See: https://creativecommons.org/licenses/by-nc-nd/4.0/.

\section{References}

1. Miller KD, Siegel RL, Lin CC, et al. Cancer treatment and survivorship statistics, 2016. CA Cancer J Clin 2016;66:271-89.

2. Mito JK, Mitra D, Barysauskas CM, et al. A comparison of outcomes and prognostic features for radiation-associated angiosarcoma of the breast and other radiation-associated sarcomas. Int J Radiat Oncol Biol Phys 2019;104:425-35.

3. Amendola BE, Amendola MA, McClatchey KD, et al. Radiation-associated sarcoma: a review of 23 patients with postradiation sarcoma over a 50-year period. Am J Clin Oncol 1989;12:411-5.

4. Kirova YM, Vilcoq JR, Asselain B, et al. Radiation-induced sarcomas after radiotherapy for breast carcinoma: a largescale single-institution review. Cancer 2005;104:856-63.

5. Petry V, Bonadio RC, Cagnacci AQC, et al. Radiotherapyinduced malignancies in breast cancer patients with TP53 pathogenic germline variants (Li-Fraumeni syndrome). Fam Cancer 2020;19:47-53.

6. Horton L, Wohlfeil M, Al-Kourainy N, et al. Rare case 
of primary leiomyosarcoma of the breast treated with wide local excision and planned cosmetic breast reduction surgery. BMJ Case Rep 2020;13:e236013.

7. Mavrogenis AF, Pala E, Guerra G, et al. Post-radiation sarcomas. Clinical outcome of 52 Patients. J Surg Oncol 2012;105:570-6.

8. Thijssens KM, van Ginkel RJ, Suurmeijer AJ, et al. Radiation-induced sarcoma: a challenge for the surgeon. Ann Surg Oncol 2005;12:237-45.

9. Cahan WG, Woodard HQ, Higinbotham NL, et al. Sarcoma arising in irradiated bone: report of eleven cases. 1948. Cancer 1998;82:8-34.

10. Mohanty SK, Balani JP, Parwani AV. Pleomorphic leiomyosarcoma of the adrenal gland: case report and review of the literature. Urology 2007;70:591.e5-7.

11. Wong LC, Huang PC, Luh SP, et al. Primary leiomyosarcoma of the nipple-areola complex: report of a case and review of literature. J Zhejiang Univ Sci B 2008;9:109-13.

12. Kamio T, Nishizawa M, Aoyama K, et al. Primary leiomyosarcoma of the breast treated by partial resection of the breast including nipple and areola: report of a case. Surg Today 2010;40:1063-7.

13. Gupta RK, Kenwright D, Naran S, et al. Fine needle aspiration cytodiagnosis of leiomyosarcoma of the breast. A case report. Acta Cytol 2000;44:1101-5.

14. Hussien M, Sivananthan S, Anderson N, et al. Primary leiomyosarcoma of the breast: diagnosis, management and

Cite this article as: Zhang W, Qu X, Fang Y. Recurrent radiation-associated leiomyosarcoma after postoperative radiotherapy for breast invasive ductal carcinoma: a case report and literature review. Ann Palliat Med 2021;10(7):8467-8473. doi: 10.21037/apm-20-2561 outcome. A report of a new case and review of literature. Breast 2001;10:530-4.

15. Olcina M, Merck B, Giménez-Climent MJ, et al. Radiation-Induced Leiomyosarcoma after Breast Cancer Treatment and TRAM Flap Reconstruction. Sarcoma 2008;2008:456950.

16. Liu Y, Wang J, Su R, et al. Postoperative radiotherapyinduced leiomyosarcoma in breast cancer: a case report and literature review. Breast Cancer 2020;27:780-4.

17. Rane SU, Batra C, Saikia UN. Primary leiomyosarcoma of breast in an adolescent girl: a case report and review of the literature. Case Rep Pathol 2012;2012:491984.

18. Sandhya B, Babu V, Parthasarathy G, et al. Primary leiomyosarcoma of the breast: A case report and review of literature. Indian J Surg 2010;72:286-8.

19. Yap J, Chuba PJ, Thomas R, et al. Sarcoma as a second malignancy after treatment for breast cancer. Int J Radiat Oncol Biol Phys 2002;52:1231-7.

20. Hayashi S, Kitada M, Matsuda Y, et al. Leiomyosarcoma arising in irradiated region after breast-conserving surgery: a case report. Surg Case Rep 2015;1:76.

21. Fujita N, Kimura R, Yamamura J, et al. Leiomyosarcoma of the breast: a case report and review of the literature about therapeutic management. Breast 2011;20:389-93.

22. Mito JK, Mitra D, Doyle LA. Radiation-Associated Sarcomas: An Update on Clinical, Histologic, and Molecular Features. Surg Pathol Clin 2019;12:139-48. 